\title{
Simulations of Deliveries of SARS-CoV-2 Positive Pregnant Women and Their Newborn Babies: Plan to Implement a Complex and Ever-Changing Protocol
}

\author{
Shantanu Rastogi, MD, MMM$M^{1,2}$ \\ ${ }^{1}$ George Washington University Hospital, Washington DC \\ ${ }^{2}$ Children's National Health Services, Washington DC \\ Am J Perinatol 2020;37:1061-1065.
}

\begin{abstract}
Address for correspondence Shantanu Rastogi, MD, MMM, George Washington University Hospital, Children's National Health System, 900 23rd Street NW, G1032 Washington, DC 20037 (e-mail: srastogi@childrensnational.org).
\end{abstract}

\begin{abstract}
Management of severe acute respiratory Syndrome corona virus-2 (SARS-CoV-2) infected pregnant women at time of delivery presents a unique challenge. The variability in the timing and the method of delivery, ranging from normal vaginal delivery to an emergent cesarean section, adds complexity to the role of the health care providers in the medical care of the patient and in the interactions, they have with other providers. These variations are further influenced by the availability of isolation rooms in the facility and adequacy of personal protective equipment. The protocols already set in place can be further challenged when the facility reaches its capacity to manage the patients.

To fulfill the goal of providing adequate management to the SARS-CoV-2 infected pregnant women and their infants, avoid variation from suggested guidelines, and decrease risk of exposure of the health care workers, the health care provider team

Keywords

- SARS-CoV-2

- COVID-19

- pregnant

- delivery

- newborn

- discharge needs to review the variations regularly. While familiarity can be achieved by reviewing the guidelines, clinical case simulations provide a more hands-on approach.

Using case-based simulations and current guidance from the Center for Disease Control, American Academy of Pediatrics, and recent reviews, we discuss a management guideline developed at our institution to facilitate provision of care to SARS-CoV2 infected pregnant women during delivery and to their infants, while protecting health care providers from exposure, and in keeping with the local facility logistics.

\section{Key Points}

- Simulation of delivery of SARS-CoV-2 positive pregnant women can minimize the risk of exposure to health care professionals.

- Four common scenarios of delivery as described can be adapted for the evolving guidelines for the management of SARS-CoV-2 positive pregnant women.

- Integrating simulations of management of SARS-CoV-2 positive pregnant women is feasible in daily clinical routine.
\end{abstract}

A large number of health care providers have been exposed to and infected by severe acute respiratory syndrome corona virus-2 (SARS-CoV-2) and some have succumbed to corona virus-induced disease 2019 (COVID-19). ${ }^{1}$ Pregnant women infected with SARS-CoV-2 present a unique challenge to health care providers at the time of delivery because of the limited time frame within which medical decisions need to be made. These decisions pertain to variation in acuity of labor, delivery received

April 21, 2020

accepted after revision

May 27, 2020

published online

July 2, 2020
Copyright $\odot 2020$ by Thieme Medical Publishers, Inc., 333 Seventh Avenue, New York, NY 10001, USA. Tel: +1(212) 760-0888.
DOI https://doi.org/ 10.1055/s-0040-1713602. ISSN 0735-1631. 
methods, and outcomes (i.e., spanning from normal vaginal delivery to emergent cesarean section). This complexity is compounded by the presence or absence of COVID-19-associated symptoms in the pregnant women as well as by the availability of health care workers, isolation rooms, and personal protective equipment (PPE). ${ }^{2}$ Although there are guidelines for specific scenarios, workflow may need to be adapted based on hospitalization of increasing numbers of SARS-CoV-2 positive patients, which may impact the capacity of the health care facility. Recognizing the need for quick adaptability, we discuss simulations developed at our institution based on current guidance from Centers of Disease Control (CDC) and American Academy of Pediatrics (AAP)..$^{3-5}$

Our facility performs universal screening on all mothers admitted in labor using reverse transcription polymerase chain reaction (RT-PCR) (Cepheid GeneXpert, Sunnyvale, CA). We then dichotomize all SARS-CoV-2 positive pregnant women admitted in labor based on the presence of symptoms. As more institutions implement universal SARS-CoV2 screening for all pregnant women in labor, ${ }^{6}$ managing simulations based on this grouping will become more important, and will need to be adapted according to the logistics of the local institution. In - Table 1, we present simulations of four major scenarios based on method and urgency of delivery among SAR-CoV-2 positive pregnant women. We then dichotomize the approach further based on the clinical assessment of the newborn. We also highlight the importance of shared decision making regarding the infant's care following its clinical assessment. For instance, further newborn care, including logistics about the infant's stay in the hospital or discharge home, will vary based on maternal testing and symptoms. Support available for the mother in the hospital and at home, ${ }^{5}$ along with the desire to feed expressed breast milk or breast feed are among the considerations we present in -Table 2. Our experience highlights the importance of routine drills to practice these different scenarios, building upon a recently published review on resuscitation and postresuscitation care of the infants born to SARS-CoV-2 positive pregnant women that outlines recommended and alternative management methods. ${ }^{7}$ The goal of simulating the different scenarios, such as those summarized in -Tables 1 and 2, is to seamlessly facilitate interdisciplinary communications and also reinforce delivery of appropriate care. We discuss one such case simulation below.

\section{Case Simulation and Debriefing}

A mother of unknown gestational age presents in advanced labor to the emergency room (ER) with unknown SARS-CoV2 status. While the obstetrician (OB1) and neonatologist (N1) (who waits outside the room with PPE ready) are called to the ER to evaluate the patient, a SARS-CoV-2 RT-PCR is sent. Since the patient is stable, she is transferred with a face mask to the obstetrics floor. She is admitted as a person under investigation in one of the negative pressure room pending the results of the RT-PCR. Per the evaluation by OB1, the patient is estimated to have full term pregnancy with growth retardation in the fetus with breech presentation. N1 discusses the possible scenarios based on type of delivery and test results with the mother over the phone. ${ }^{3-5,7}$ Obstetrics, anesthesia, and the neonatal team (N1 and a neonatal nurse [NN1]) along with the hospital-designated safety officer (who observes all the providers as they don and doff in a prescribed manner, ${ }^{8}$ and follow infection control protocols) huddle to plan for vaginal delivery and possible cesarean section if there is failure to progress or if there is fetal distress. Decision is made to proceed with epidural administration and a trial for a vaginal delivery. The baby delivers vaginally. As per the third column of - Table 1, NN1 receives the baby and places it in a heated isolette outside the negative pressure room and doffs with the help of safety officer. N1 covers the isolette with a plastic sheet and takes the baby to the isolation room in the neonatal intensive care unit (NICU), which is in the same corridor. We recognize that the physical structure of the hospital allows us to do this in less than 30 seconds. In facilities where the NICU is at a distance, the baby may need to be moved to another negative pressure labor room. Alternatively, N1 may enter the labor room after delivery with the radiant warmer placed at least 6 feet away from the mother, and additionally separated by a physical barrier, such as a plastic curtain. ${ }^{7}$ Following transfer to the NICU, the infant is taken to the isolation room, where $\mathrm{N} 1$ and a neonatal nurse (NN2) will evaluate the baby. After stabilization and anthropometric measurements, the baby is given a bath. In the interim, the results from the mother's testing reveal that she is positive for SARS-CoV-2. She is updated on the results, and since there is no significant other with her, she is presented with two alternative methods of providing care of the infant. She decides to have the infant in the room, feeds the infant mother's own milk, and follows directions to keep the baby 6 feet away, with appropriate use of mask and hand and breast hygiene. The infant is tested in 24 hours by a SARS-CoV-2 RT-PCR which came out negative. As per the mother's request, the dyad is discharged at approximately 36 hours after all routine newborn screenings are completed. The mother is instructed about the needed precautions, ${ }^{5}$ as she lives alone and has no support at home. In addition, a detailed report is sent to the pediatrician by a fax or directly updating them with a phone call. Having running this simulation, we then discuss issues that may have arisen and the many variations in presentation or decision-making which may have occurred as further drills. The drills typically occur after the morning and evening sign outs (as teams work in 12 or 24 hours shifts). Each drill takes approximately 15 to 20 minutes, at which time each participant is given a small card which lists the exact task that the person has to perform in the varied case scenarios. These simulations reinforce management as well as introduce and clarify each participant's role, thereby decreasing the likelihood of miscommunication, which is an important cause of poor clinical outcomes and breach in infection control protocols. The improved communication during these simulations also decreases the health care providers' anxiety regarding exposure as they become aware of and comfortable with their roles and variations in the role that may occur 

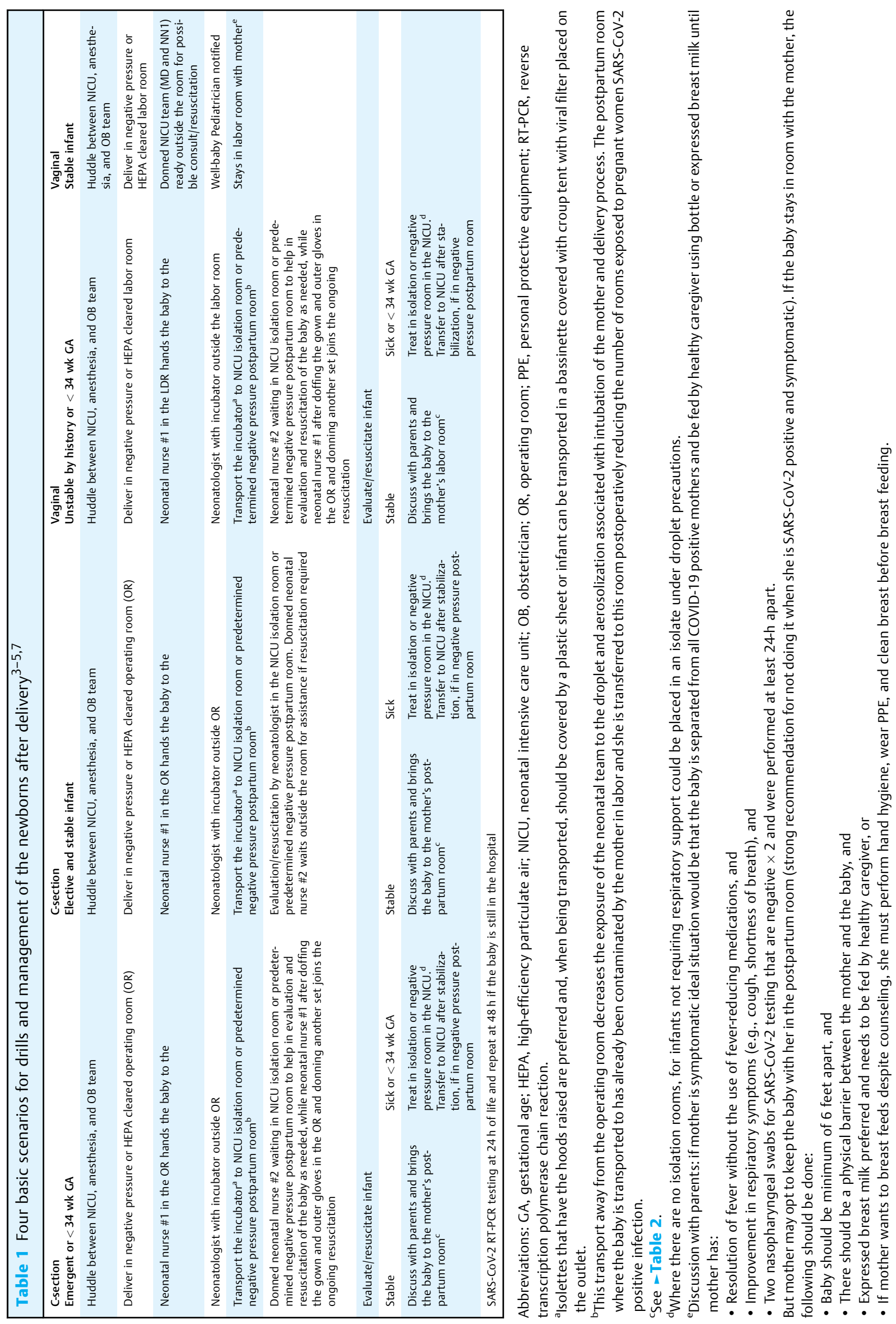

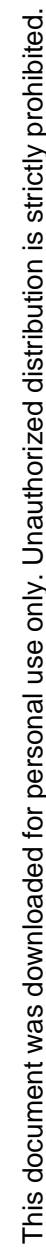


Table 2 Plan for discharge of the baby based on shared decision between the health care providers and the parents ${ }^{5}$

Scenario Ideal (test-based strategy)

Mother positive BUT asymptomatic. Infant is negative

Mother is positive AND symptomatic. Infant is negative

Mother is positive BUT asymptomatic. Infant is positive BUT asymptomatic

Mother is positive WITH or WITHOUT symptoms.

Infant is positive AND symptomatic healthy caregiver specimens) 60 years of age specimens) infected persons after birth of respiratory symptoms

AND
The newborn would be discharged to a

Mother has negative results of an FDA Emergency Use Authorized COVID-19 molecular assay for detection of SARSCoV-2 RNA from at least two consecutive respiratory specimens collected $\geq 24 \mathrm{~h}$ apart (total of two negative

The newborn could be transferred to a healthy caregiver preferably below

Mother has negative results of an FDA Emergency Use Authorized COVID-19 molecular assay for detection of SARSCoV-2 RNA from at least two consecutive respiratory specimens collected $\geq 24 \mathrm{~h}$ apart (total of two negative

Infants should not be cared for by un-

The newborn would be discharged to a mother who remains on contact and droplet precautions until mother has: - has negative results of FDA Emergency Use Authorized COVID-19 molecular assay for detection of SARS-CoV-2 RNA from at least two consecutive respiratory specimens collected $\geq 24 \mathrm{~h}$ apart (total of two negative specimens) Infants determined to be positive by testing (or whose status cannot be determined due to lack of testing), but with no symptoms of COVID-19, may be discharged home on a case-by-case basis with appropriate precautions and plans for planned and frequent outpatient follow-up contacts (either by phone, telemedicine, or in office) through $14 \mathrm{~d}$

Infant remains hospitalized until at least $3 \mathrm{~d}(72 \mathrm{~h})$ have passed since improvement

until there are two consecutive negative tests for SARS-CoV-2 of combined throat/nasopharynx specimens done at 48- to 72-h intervals, first done at $24 \mathrm{~h}$ of life Symptomatic mothers with COVID-19 should not visit infants requiring neonatal intensive care until they meet symptom based or test based requirements Symptom based:

1. Resolution of fever without the use of antipyretics for at least $72 \mathrm{~h}$ and improvement (but not full resolution) in respiratory symptoms AND

2. At least $10 \mathrm{~d}$ have passed since symptoms first appeared OR

Test based:

1. Negative results of FDA Emergency Use Authorized COVID-19 molecular assay for detection of SARS-CoV-2 RNA from at least two consecutive respiratory specimens collected $\geq 24 \mathrm{~h}$ apart (total of two negative specimens)

Abbreviations: FDA, Food and Drug Administration; RNA, ribonucleic acid; RT-PCR, reverse transcription polymerase chain reaction.

Note: All infants born to SARS-CoV-2 positive women are discharged after detailed report to the pediatrician to prevent the mother coming for the appointment and exposing the clinic staff. Advice is given to the caregiver or the mother to take the baby to the clinic by a healthy member of the family and subsequent daily follow-up done by phone, telemedicine, or in office. Repeat SARS-CoV-2 RT-PCR may be performed at 48 hours if baby discharged before 48 hours from the hospital. 
as the clinical scenarios evolve. We have also observed that instituting these simulations has decreased the instances of conflicting information that parents receive from different caregivers, which has directly improved patient satisfaction, even among the most anxious patients. The effectiveness of our approach in managing variability in clinical presentation and thereby management plans until the time of delivery, highlights the advantages of creating a similar document suited to the local institutions. In addition to the guidelines, routine simulation by the multidisciplinary health care team consisting of obstetricians, anesthesiologists, neonatologists, nurses, and the operating room technicians will improve medical outcomes while reducing inadvertent exposure of the health care providers to SARS-CoV-2 positive mothers. ${ }^{9}$ Our simulations add to a recent publication limited to the second stage of labor, where the authors demonstrated that development of a multidisciplinary management plan decreases the exposure of health care personnel to SARSCoV-2. ${ }^{10}$ We would also like to highlight that, in light of the evolving knowledge on this new virus, the scenarios should be constantly updated to incorporate new guidance from the CDC, AAP, and other subspecialty societies. For instance, there has been an increase in deliveries of SARS-CoV-2 positive pregnant women outside the hospital and emergent delivery may also occur in the ER or in the medical intensive care unit, where a pregnant woman with COVID-19 may be hospitalized. These approaches also need the support of clinical leadership, such as division directors, so that everyone is encouraged to participate and there are no missing links that could lead to poor patient care and/or increased risk of exposure of the health care providers.

It is evident that this health care crisis will not completely disappear in the near future. Continuing such simulations, even when the incidence of SARS-CoV-2 positivity among pregnant women is lower, will help keep health care providers in a state of readiness for appropriate management of these deliveries, ensuring that the health care providers remain protected from unnecessary exposure and infection while continuing to provide the best outcomes.

Conflict of Interest

None declared.

\section{References}

1 Li R, Chen X, Wang Y, et al. Risk factors of healthcare workers with corona virus disease 2019: A retrospective cohort study in a designated hospital of Wuhan in China. Clin Infect Dis 2020;2020. Doi: 10.1093/cid/ciaa287Epub

2 Wang J, Zhou M, Liu F. Reasons for healthcare workers becoming infected with novel coronavirus disease 2019 (COVID-19) in China. J Hosp Infect 2020;105(01):100-101

3 Considerations for Inpatient Obstetric Healthcare Settings. Available at: https://www.cdc.gov/coronavirus/2019-ncov/hcp/inpatient-obstetric-healthcare-guidance.html. Accessed May 24, 2020

4 Puopolo KM, Hudak ML, Kimberlin DW, Cummings J. Initial guidance: management of infants born to mothers with COVID19. Available at: https://downloads.aap.org/AAP/PDF/COVID-19\% 2019\%20Initial\%20Newborn\%20Guidance.pdf. Accessed May 24, 2020

5 Discontinuation of Isolation for Persons with COVID-19 Not in Healthcare Settings (Interim Guidance). Available at: https:// www.cdc.gov/coronavirus/2019-ncov/hcp/disposition-in-homepatients.html. Accessed May 24, 2020

6 Sutton D, Fuchs K, D’Alton M, Goffman D. Universal screening for SARS-CoV-2 in women admitted for delivery. N Engl J Med 2020; 382(22):2163-2164

7 Chandrasekharan P, Vento M, Trevisanuto D, et al. Neonatal resuscitation and post resuscitation care of infants born to others with suspected or confirmed SARS-CoV-2 infection. Am J Perinatol 2020. Doi: 10.1055/s-0040-1709688

8 For donning and doffing of PPE. Available at: https://www.cdc.gov/ coronavirus/2019-ncov/hcp/using-ppe.html. Accessed May 24, 2020

9 Riley W, Davis S, Miller K, Hansen H, Sainfort F, Sweet R. Didactic and simulation nontechnical skills team training to improve perinatal patient outcomes in a community hospital. Jt Comm J Qual Patient Saf 2011;37(08):357-364

10 Palatnik A, McIntosh JJ. Protecting labor and delivery personnel from COVID-19 during the second stage of labor. Am J Perinatol 2020. Doi: $10.1055 / \mathrm{s}-0040-1709689$ 